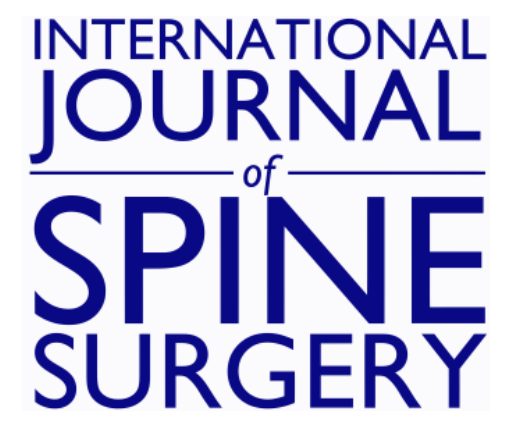

\title{
Effect of TLIF Cage Placement on In Vivo Kinematics
}

Alejandro D. Castellvi, Shankar K. Thampi, Daniel J. Cook, Matthew S. Yeager, Yuan Yao, Qing Zou, Donald M. Whiting, Michael Y. Oh, Edward R. Prostko and Boyle C. Cheng

Int J Spine Surg 2015, 9 ()

doi: https://doi.org/10.14444/2038

http://ijssurgery.com/content/9/38

This information is current as of April 26, 2023.

Email Alerts Receive free email-alerts when new articles cite this article. Sign up at:

http://ijssurgery.com/alerts 


\section{Effect of TLIF Cage Placement on In Vivo Kinematics}

Alejandro D. Castellvi, MD, ${ }^{1}$ Shankar K. Thampi, BS, ${ }^{2}$ Daniel J. Cook, MS, ${ }^{1}$ Matthew S. Yeager, BS, ${ }^{2}$ Yuan Yao, MD, ${ }^{3}$ Qing Zou, MD, ${ }^{3}$ Donald M. Whiting, MD,1,2 Michael Y. Oh, MD,1,2 Edward R. Prostko, MD,1,2 Boyle C. Cheng, PhD1,2

${ }_{1}^{1}$ Department of Neurosurgery, Allegheny Health Network, Pittsburgh, Pennsylvania, ${ }^{2}$ Drexel University College of Medicine, Philadelphia, Pennsylvania, ${ }^{3}$ No.117 Hospital of the People's Liberation Army, Hangzhou, China

\section{Abstract}

\section{Background}

The influence of interbody cage positioning on clinical outcomes following lumbar interbody fusion is not well understood, though it has been hypothesized to play a significant role in stability of the treated level. The purpose of this study was to evaluate any correlations between cage placement in TLIF procedures and post-operative kinematics.

\section{Methods}

Thirteen patients who had previously undergone a TLIF procedure were evaluated using the Vertebral Motion Analysis (VMA) system, an automated fluoroscopic method of tracking kinematics in vivo. Upright and recumbent bending platforms were used to guide patients through a set range of motion (ROM) standing up and lying down, respectively, in both flexion-extension (FE) and lateral bending (LB). Intervertebral ROM was measured via fluoroscopic images captured sequentially throughout the movement. DICOM images acquired by the VMA system were used to calculate cage positioning. Intra-rater and inter-rater reliability of TLIF cage position were also assessed.

Results

Statistically significant correlations were noted between sagittal cage position and lying LB $(r=-0.583, p=0.047)$, and coronal cage positioning with both standing $(\mathrm{r}=0.672, \mathrm{p}=0.012)$ and lying $\mathrm{LB}(\mathrm{r}=0.632, \mathrm{p}=0.027)$. Additionally, the correlation between sagittal cage position and standing FE was trending towards significance $(\mathrm{r}=$ $-0.542, \mathrm{p}=0.055)$.

\section{Conclusions}

The intuitive correlation between coronal cage position and both standing and lying lateral bending ROM is supported by the data from this study, suggesting placement closer to midline is optimal for stability. Additionally, the VMA system appears to be a sensitive and repeatable means to obtain information on postoperative kinematic outcomes. Further work to establish the relationship between cage placement, these kinematic outcomes and, potentially, functional pain outcomes seems to be warranted based on the results obtained here.

KEYWORDS: TRANSFORAMINAL LUMBAR INTERBODY FUSION, VERTEBRAL MOTION ANALYSIS, CAGE POSITIONING, STABILITY

Volume 9 ARTICLE 38 - BIOMECHANICS SPECIAL ISSUE DOI: 10.14444/2038

\section{Introduction}

The concept of lumbar interbody fusion was initially developed by authors including Capener and Mercer in the early 1930's as an alternative to the standard posterior arthrodesis procedures of the period. Both men argued that from a biomechanical standpoint, posterior union via the spinous processes was not an effective technique due to the significant loading on the graft, and advocated using an anterior graft to fix the vertebral bodies instead. ${ }^{1,2}$ Early studies compared posterior spinal fusion with and without an in-

Downloaded from http://ijssurgery.com/ by guest on April 26, 2023 terbody device, observing that the interbody group had higher rates of fusion and lower rates of hardware complications secondary to biomechanical issues. ${ }^{3}$ Since then, numerous approaches to lumbar interbody fusion have been developed, including anterior (ALIF), posterior (PLIF), lateral, and transforaminal (TLIF). PLIF was popularized by Cloward, who emphasized that it not only maintained the intervertebral space and foramen while restricting movement, but also avoided the morbidity associated with exposure during ALIF procedures. ${ }^{3,4}$ 
Complications were still noted with the posterior approach however, including damage to the cauda equina and nerve roots during manipulation, increased incidence of durotomy, and loss of stability secondary to damage of the facet joints. ${ }^{3,5}$ To address these problems, Harms and Rolinger devised a procedure utilizing a unilateral transforaminal approach with pedicle fixation, which minimized retraction of the thecal sac and did not require destruction of the contralateral pars, lamina, and facet. ${ }^{5}$ Additionally, TLIF was shown to be superior to PLIF in reestablishing proper lumbar lordosis and decompressing the neural foramina. ${ }^{3,5}$ These advantages have prompted many surgeons to transition to the transforaminal approach.

Numerous factors affect stability of the FSU, including patient-related properties such as bone density and implant-related characteristics such as cage surface area, shape, and operative technique. Previous publications have suggested that ideally the graft area should cover at least $30-40 \%$ of the vertebral body endplate. ${ }^{6}$ The influence of interbody cage positioning on in vivo biomechanics following lumbar interbody fusion is not well understood. It is likely that the location, position, and shape of the interbody fusion mass may influence the stability of the treated level(s). This variability in structural stability may correlate to variability in treatment outcomes in terms of recurrence of symptoms, rate of reoperation, prevalence of pseudoarthrosis, or development of adjacent level disease. Due to the small operative window, asymmetry of the approach, and relatively small surface area or "footprint" of the cage, the TLIF procedure may be particularly susceptible to problems associated with cage positioning. Limited in vitro biomechanical and finite element analyses have been conducted to investigate the influence of cage positioning on the structural stability of the functional spinal unit (FSU). While some have found that positioning of the graft has no bearing on stability, ${ }^{5,-9}$ others have found that an anteriorly placed graft imparts the construct with greater stability and produces less strain on the posterior elements and instrumentation..$^{10-12}$ Due to the limited in vivo data available, it is apparent that more information needs to be obtained in order to gain a better understanding of the biomechanical effects of cage positioning.
The primary objective of this study was to assess correlations between cage placement in TLIF procedures and post-operative kinematics. Cage placement was assessed via post-operative fluoroscopic imaging while kinematic evaluation was conducted using the Vertebral Motion Analysis (VMA) system, a new highly reliable and repeatable computerized tracking method utilizing fluoroscopy.

\section{Materials and Methods}

\section{Patient Selection}

Thirteen patients, both male and female, age 18 years or over who had previously undergone a TLIF procedure were selected for this study. Informed consent was obtained prior to participation as approved by the Institutional Review Board. All patients were implanted with Capstone (Medtronic, Memphis, TN) polyetheretherketone (PEEK) TLIF straight cages via standard surgical technique.

\section{Vertebral Motion Analysis System}

Imaging was obtained utilizing the Vertebral Motion Analysis (VMA) system, which employs upright and recumbent patient bending platforms and a standard surgical C-Arm. Each bending platform consisted of a radiolucent disc which acted as the center of rotation. The upright platform guided active lumbar bending under the weighted condition, while constricting flexion-extension (FE) to the sagittal plane. The recumbent platform controlled passive lumbar bending, which minimized the gravitational and muscular forces that are present during standing radiographs. Trunk bending was completed to a predetermined total range of motion (ROM) of $70^{\circ}$. In recumbent motion, the $\mathrm{FE}$ angles spanned a range of $\pm 35^{\circ}$, while in upright motion the ROM presets were adjusted to allow for $20^{\circ}$ of extension and $50^{\circ}$ of flexion. This compensated for the reduced capability of lumbar extension resulting from extended hips in an erect posture.

Flexion and extension were captured via fluoroscopy as separate sequences, beginning in the neutral position, progressing to the maximum angle, and then returning to neutral. The automated tracking process consisted of a template based approach using normalized, mean-centered cross-correlation as a matching 
criterion to automatically identify each vertebra throughout the image sequence. This method has been previously described ${ }^{13,14}$ and analysis of repeatability of the VMA system in comparison to manual measurements of intervertebral motion has been previously conducted. In that study, the intra-rater and inter-rater coefficient of repeatability (CR) for VMA was found to be $2.20 \%$ and $3.90 \%$, respectively, compared to $11.75 \%$ and $12.43 \%$ for manual. ${ }^{15}$

Only measurements at neutral and maximum patient bending angles were used for assessment. Overall intervertebral ROM was calculated for the treatment level of each patient in standing and lying FE and lateral bending (LB).

\section{Measurement of TLIF Cage Positioning}

The raw DICOM images acquired by the VMA system were used for calculation of cage positioning. The inferior margins of the vertebral body superior to the cage and the superior margins of the vertebral body inferior to the cage were chosen as the landmarks to define an anatomical reference frame in both the sagittal and coronal plane. Landmarks were identified in ImageJ (National Institutes of Health, Bethesda, MD) as shown in Figure 1, and these coordinates were exported to text for analysis in MATLAB (MathWorks, Natick, MA). The origin of the coordinate system in each plane (shown as a red dot in Figure 1) was defined as the centroid of the four anatomical landmarks defining the vertebral body borders in that plane. In the sagittal plane, the positive $\mathrm{x}$-axis (shown as the solid vector in Figure 1) of the coordinate system was defined in the direction of a vector connecting the two inferior vertebral body landmarks oriented anteriorly (shown as the dashed line in Figure 1). In the coronal plane, the positive $x-$ axis was defined in the direction of the vector connecting the two inferior vertebral body landmarks oriented to the right. The centers of each of the three radiopaque markers embedded within the cage were used as landmarks for identifying cage location. The center of the cage was defined as the midpoint of the line connecting the posterior cage landmark and the midpoint of the two anterior cage landmarks. The location of the cage centroid along the $\mathrm{x}$-axis was calculated relative to the intervertebral space centroid and normalized to vertebral body depth for the sagit- tal plane or width for the coronal plane, based on the distance between the inferior vertebral body landmarks. Cage positioning will hereafter be expressed as a percentage of the respective vertebral body dimension. "Sagittal plane location" will be used to refer to the anterior-posterior position of the cage, and "coronal plane location" will be used to refer to the lateral position of the cage. Considering that, depending on patient presentation, treatment may be pursued via the left or the right transforaminal route, the absolute value of the distance from the origin in the coronal plane was used to assess coronal plane location in order to address discrepancies due to direction of approach.

\section{Reliability of TLIF Cage Position Measurements}

A reliability analysis was conducted on the measurement of TLIF cage positioning. Two observers identified landmarks based on the algorithm described above twice for each of the patients in the study. The normalized position of the TLIF cage relative to the defined intervertebral space was calculated based on each set of measurements made by each observer. The Bland-Altman method was used to establish repeatability and reliability statistics for the measurement algorithm. The differences between the first and second round measurements for each observer were used to assess intra-rater reliability, while the

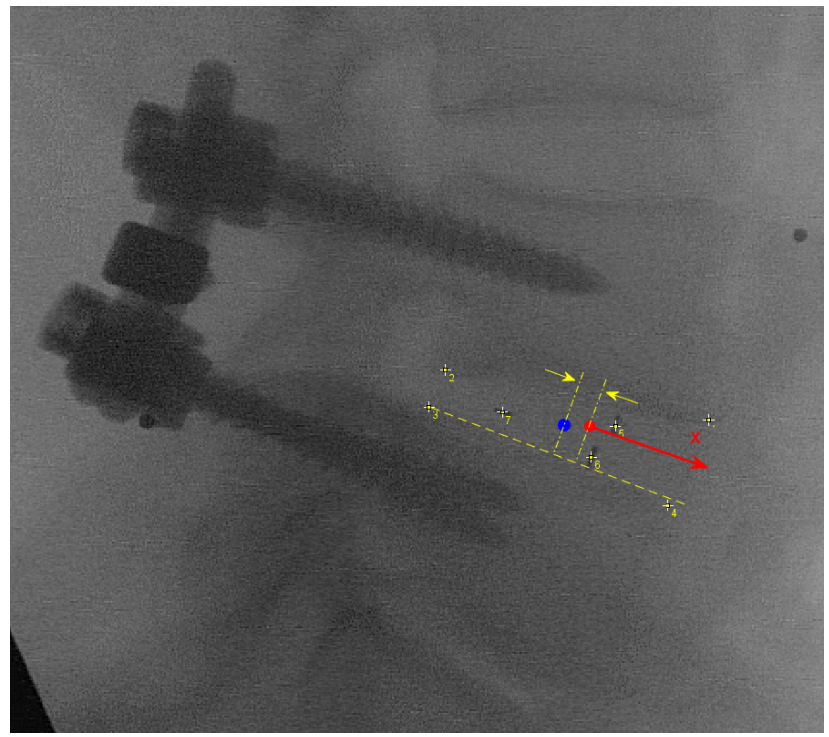

Fig. 1. Illustration of Anterior-Posterior Position of the Spacer within the Interbody Space (Vertebral Landmarks = points 1-4; Spacer Landmarks = points 5-7; X-axis - parallel to dashed line connecting points 3 and 4; Center of Interbody Space = Red Dot; Center of Spacer = Blue Dot; Measured distance along $\mathrm{X}$-axis is indicated by the arrows). 
differences between the two observers' average first and second round measurements were used for interrater reliability. The coefficient of repeatability (CR) was calculated as twice the standard deviation (SD) of paired differences for each inter- and intra-rater analysis.

\section{Results}

All data was successfully obtained for each of the patients enrolled with the exception of the Lying LB kinematics of a single patient due to the oblique positioning of the intervertebral level relative to the imaging plane.

\section{Inter-rater and Intra-rater Reliability}

The intra-rater CR for observers 1 and 2 were $3.4 \%$ and $3.7 \%$ in the sagittal plane and $5.3 \%$ and $5.7 \%$ in the coronal plane, respectively. The inter-rater $\mathrm{CR}$ was $4.9 \%$ in the sagittal plane and $5.6 \%$ in the coronal plane. The mean of the two observers' first round measurements were used in the analysis for this study.

\section{Cage Position Descriptive Statistics}

Table 1 lists the operative level and the measured cage positions for each patient enrolled in the study. Nine of the patients in the study received left-sided TLIF while the remaining four received right-sided TLIF. Table 2 provides the descriptive statistics for the sagittal and coronal plane TLIF cage measurements across all patients enrolled.

\section{Correlation Results}

All statistical analysis was conducted in SPSS (IBM, Armonk, NY). Correlation coefficients were calculated between cage position and ROM metrics. The absolute value of cage position in the coronal plane was used to account for direction of approach. These values are shown in Table 3. Statistically significant correlations were based on $\mathrm{p}<0.05$, while statistically trending correlations were based on $0.05 \mathrm{p}<0.10$. Scatter plots of significant and trending correlations are provided in Figure 2, Figure 3, Figure 4, \& Figure 5.

The significant positive correlation between Standing and Lying LB ROM and coronal cage position ( $p$
$=0.012$ and $\mathrm{p}=0.027$, respectively) seen in Figure 3 and Figure 4 strongly suggests that there is increased stability the closer the cage is placed to midline, as is expected. Additionally, the trending negative correlation $(\mathrm{p}=0.055)$ between Standing FE ROM and sagittal plane cage position seen in Figure 1 is intuitive in that a cage placed further toward the anterior margin of the intervertebral space would provide for a more stable construct. On the other hand, the significant negative correlation ( $\mathrm{p}=0.047$ ) between Lying LB ROM and sagittal cage position seen in Figure 2 is slightly less intuitive, as one would expect anterior cage positioning to correlate with FE ROM and not necessarily LB ROM. However, it appears that anterior cage positioning may provide greater stability in LB.

\begin{tabular}{|c|c|c|c|c|}
\hline Patient & $\begin{array}{r}\text { Intervertebral } \\
\text { Level }\end{array}$ & $\begin{array}{l}\text { Sagittal } \\
\text { Position }\end{array}$ & $\begin{array}{l}\text { Coronal } \\
\text { Position }\end{array}$ & $\begin{array}{r}\text { Days } \\
\text { Post-Op }\end{array}$ \\
\hline 1 & L4-L5 & $-1.85 \%$ & $-6.83 \%$ & 143 \\
\hline 2 & L4-L5 & $-2.77 \%$ & $11.93 \%$ & 143 \\
\hline 3 & L4-L5 & $-8.48 \%$ & $10.94 \%$ & 172 \\
\hline 4 & L4-L5 & $-0.52 \%$ & $-1.28 \%$ & 152 \\
\hline 5 & L5-S1 & $9.80 \%$ & $8.78 \%$ & 66 \\
\hline 6 & L4-L5 & $-2.49 \%$ & $-7.04 \%$ & 159 \\
\hline 7 & L2-L3 & $-3.14 \%$ & $11.67 \%$ & 148 \\
\hline 8 & L4-L5 & $-8.20 \%$ & $3.90 \%$ & 66 \\
\hline 9 & L4-L5 & $-4.01 \%$ & $-10.42 \%$ & 256 \\
\hline 10 & L4-L5 & $2.04 \%$ & $6.15 \%$ & 337 \\
\hline 11 & L3-L4 & $-4.58 \%$ & $20.39 \%$ & 192 \\
\hline 12 & L4-L5 & $-3.52 \%$ & $3.75 \%$ & 295 \\
\hline 13 & L5-S1 & $-2.11 \%$ & $3.42 \%$ & 913 \\
\hline
\end{tabular}

\begin{tabular}{|c|c|c|c|}
\hline & \multicolumn{2}{|c|}{ Sagittal Position } & Coronal Position \\
\hline Mean & & $-2.29 \%$ & $8.19 \%$ \\
\hline Median & & $-2.77 \%$ & $7.04 \%$ \\
\hline \multirow{3}{*}{ Percentiles } & 25 & $-4.29 \%$ & $3.83 \%$ \\
\hline & 50 & $-2.77 \%$ & $7.04 \%$ \\
\hline & 75 & $-1.18 \%$ & $11.31 \%$ \\
\hline
\end{tabular}


Table 3. Correlation Coefficients.

\begin{tabular}{|c|c|c|c|}
\hline & & $\begin{array}{l}\text { Sagittal } \\
\text { Position }\end{array}$ & $\begin{array}{l}\text { Coronal } \\
\text { Position }\end{array}$ \\
\hline \multirow{3}{*}{ Standing FE } & Pearson Correlation & -0.542 & 0.319 \\
\hline & $\begin{array}{l}\text { 2-Tailed } \\
\text { Significance }\end{array}$ & 0.055 & 0.289 \\
\hline & $\mathbf{N}$ & 13 & 13 \\
\hline \multirow{3}{*}{ Lying FE } & Pearson Correlation & 0.439 & -0.191 \\
\hline & $\begin{array}{l}\text { 2-Tailed } \\
\text { Significance }\end{array}$ & 0.134 & 0.533 \\
\hline & $\mathbf{N}$ & 13 & 13 \\
\hline \multirow{3}{*}{$\begin{array}{l}\text { Standing } \\
\text { LB }\end{array}$} & Pearson Correlation & -0.324 & $0.672^{*}$ \\
\hline & & 0.280 & 0.012 \\
\hline & & 13 & 13 \\
\hline \multirow{3}{*}{ Lying LB } & Pearson Correlation & $-0.583^{*}$ & $0.632 *$ \\
\hline & $\begin{array}{l}\text { 2-Tailed } \\
\text { Significance }\end{array}$ & 0.047 & 0.027 \\
\hline & $\mathbf{N}$ & 12 & 12 \\
\hline
\end{tabular}

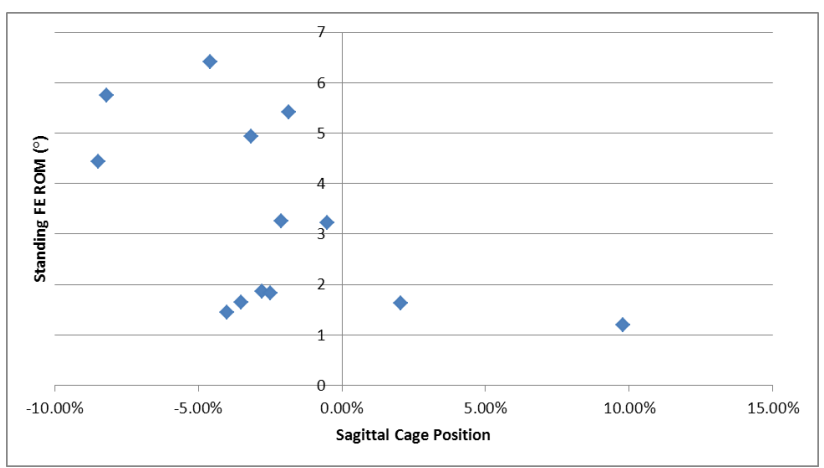

Fig. 2. Standing FE ROM vs. Sagittal Cage Position $(r=-0.542, p=$ $0.055)$.

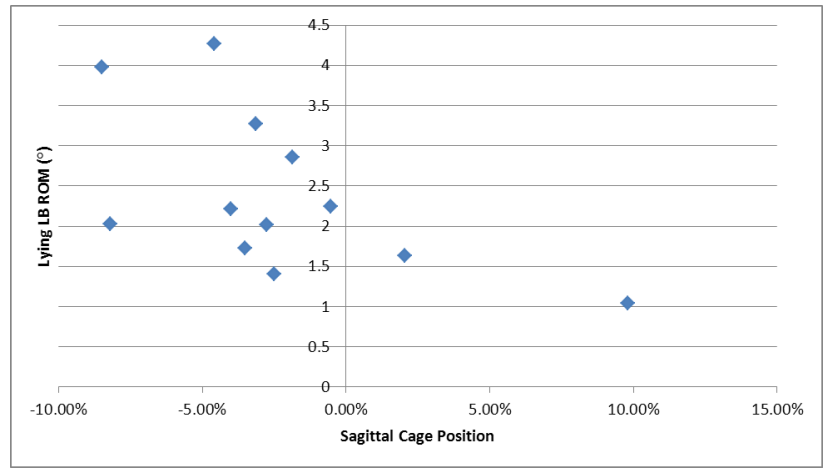

Fig. 3. Lying LB ROM vs. Sagittal Cage Position $(r=-0.583, p=0.047)$.

\section{Discussion}

While several in vitro studies investigating the relative merits of various interbody cage designs in terms of stability and subsidence are described in the literature ${ }^{16}$ very little has been done to establish optimum cage placement. Quigley et al investigated differences in strain and cyclic failure given three interbody cage locations: anterior, middle and anterolateral. ${ }^{10}$ While the group showed greater strain on posterior hardware and faster load to failure for the anterolateral cage position, extrapolation to the clinical scenario is difficult because the study relied on polyurethane blocks as vertebral analogs and axial compression as its loading mode. To assess the feasibility of measuring TLIF cage position and its relation to intervertebral kinematics, a retrospective study of patients who had undergone TLIF surgery was conducted.

When considering the three-joint complex of the FSU, the interbody cage provides support of anterior column loading following discectomy, and thus plays a defining role in initial stability until arthrodesis is achieved. Given the asymmetry of the TLIF construct, it is reasonable then to expect that greater sta-

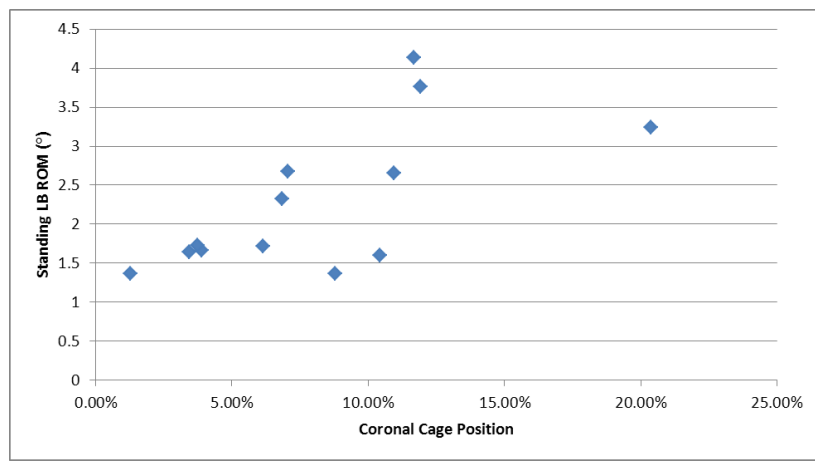

Fig. 4. Standing LB ROM vs. Coronal Cage Position $(r=0.672, p=0.012)$

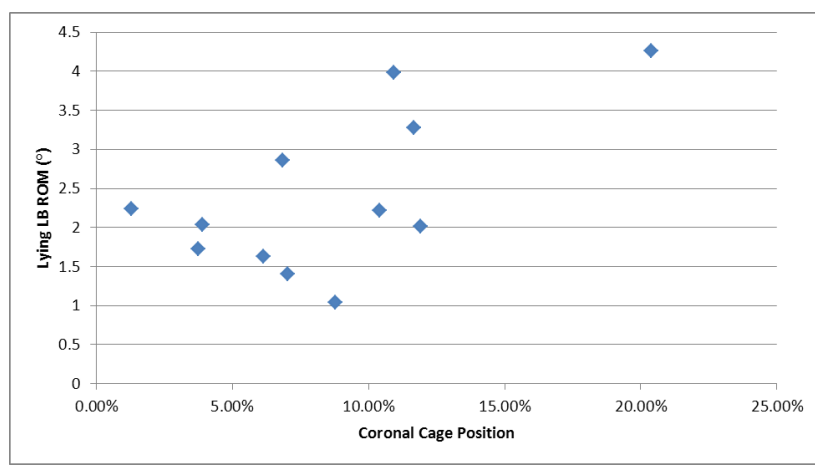

Fig. 5. Lying LB ROM vs. Coronal Cage Position $(r=0.632, p=0.027)$.

Downloaded from http://ijssurgery.com/ by guest on April 26, 2023 
bility would be achieved with cage placement near the vertebral body midline. This corresponds well to the findings of this study, which demonstrate that in TLIF procedures placing the cage closer to midline in the coronal plane provides optimal stability in lateral bending, with or without the effects of an axial load.

Additionally, the data suggests placing the cage further anterior in the sagittal plane makes for a more stable construct in flexion and extension, as would be intuitively expected. Placing the cage further from the posterior hardware would tend to provide a more stable construct. This correlates well with previously published literature based on in vitro studies. ${ }^{10-12}$ Based on interpretation of the associated scatter plots, it appears that cage location anteriorly in the sagittal plane may also limit ROM in LB, however it is difficult to draw definitive conclusions from this information.

\section{Future Work}

Further work to establish the relationship between cage placement, kinematic outcomes such as those evaluated in this study and, potentially, functional pain outcomes seems to be warranted based on the results obtained here, which provide preliminary support for the hypothesis that cage position can significantly affect mechanical clinical outcomes. A limitation of this study is the lack of control placed on the time of post operative analysis as subjects ranged from 66 to 913 days post operatively (shown in Table1). Additionally, no evaluation of fusion was conducted as a part of this study. A prospective study with a larger sample size and in which such variables such as cage placement, cage size, treatment level, surgical orientation (i.e. left vs. right TLIF) and length to radiographic follow-up can be controlled may provide greater insight into optimum cage placement. Outcome measures such as pain scores and rates of reoperation could be used for assessment. An ideal study design may involve navigated TLIF placement to predefined target zones in randomized patient groups, followed by VMA and pain score follow up at regular intervals. Given the wide variety of potential target zones and orientations that could be defined within the interbody space and the subsequent effect on the sample size required to conduct the clinical study, the appropriate discretization of the disc space may require a preliminary biomechanical study or compelling surgical rationale.

Additionally, it should be noted that the results here are limited to TLIF type cages. Due to their relatively small surface area, the positioning of intervertebral cages in TLIF procedures appears to play a significant role in post-operative stability. However, this may not be true of larger interbody devices, such as those used in ALIF procedures.

\section{References}

1. Capener N. Spondylolisthesis. Br J Surg. 1932;19(75):374-386.

2. Mercer W. Spondylolisthesis. Am J Surg. 1939;43(2):367-378.

3. Reames D, Smith J, Shaffrey C. Posterior and Transforaminal Lumbar Interbody Fusion. In: Benzel E, ed. Spine Surgery: Techniques, Complication Avoidance, and Management. Vol 1. Philadelphia, PA: Elsevier Saunders; 2012:513-522.

4. Cloward R. The treatment of ruptured lumbar intervertebral discs by vertebral body fusion. $J$ Neurosurg. 1953;10:154-168.

5. Ames C, Acosta F, Chi J, et al. Biomechanical Comparison of Posterior Lumbar Interbody Fusion and Transforaminal Lumbar Interbody Fusion Performed at 1 and 2 Levels. Spine (Phila Pa 1976). 2005;30(19):E562-E566.

6. Closkey R, Parsons J, Lee C, Blacksin M, Zimmerman M. Mechanics of Interbody Spinal Fusion: Analysis of Critical Bone Graft Area. Spine (Phila Pa 1976). 1993;18(8):1011-1015.

7. Tencer A, Hampton D, Eddy S. Biomechanical properties of threaded inserts for lumbar interbody spinal fusion. Spine (Phila Pa 1976).

1995;20(22):2408-2014.

8. Wang S, Goel V, Fu C, et al. Posterior instrumentation reduces differences in spine stability as a result of different cage orientations: an in vitro study. Spine (Phila Pa 1976). 2005;30(1):62.

9. Faundez A, Mehbod A, Wu C, Wu W, Ploumis A, Transfeldt E. Position of interbody spacer in transforaminal lumbar interbody fusion: effect on 
3-dimensional stability and sagittal lumbar contour. $J$ Spinal Disord Tech. 2008;21:175-180.

10. Quigley KJ, Alander DH, Bledsoe JG. An in vitro biomechanical investigation: variable positioning of leopard carbon fiber interbody cages. J Spinal Disord Tech. Aug 2008;21(6):442-447.

11. Kwon B, Berta S, Daffner S, et al. Radiographic analysis of transforaminal lumbar interbody fusion for the treatment of adult isthmic spondylolisthesis. J Spinal Disord Tech. 2003;16(5):469-476.

12. Bono CM, Khandha A, Vadapalli S, Holekamp S, Goel VK, Garfin SR. Residual sagittal motion after lumbar fusion: a finite element analysis with implications on radiographic flexion-extension criteria. Spine (Phila Pa 1976). Feb 15 2007;32(4):417-422.

13. Muggleton JM, Allen R. Automatic location of vertebrae in digitized videofluoroscopic images of the lumbar spine. Med Eng Phys. Jan 1997;19(1):77-89.

14. Breen AC, Muggleton JM, Mellor FE. An objective spinal motion imaging assessment (OSMIA): reliability, accuracy and exposure data. BMC Musculoskelet Disord. 2006;7:1.

15. Yeager M, Cook D, Cheng B. Reliability of computer-assisted lumbar intervertebral measure- ments using a novel vertebral motion analysis system. Spine J. 2014;14(2):274-281.

16. Oxland TR, Lund T. Biomechanics of standalone cages and cages in combination with posterior fixation: a literature review. Eur Spine J. Feb 2000;9 Suppl 1:S95-101.

\section{Disclosures}

This study was funded in part by Medtronic.

Michael Oh is a consultant for Aesculap and is on the speakers bureau for St. Jude Medical, Inc. All other authors declare no relevant disclosures.

\section{Corresponding Author}

Boyle C. Cheng, 420 East North Ave, Suite 302, Pittsburgh, PA 15212. boylecheng@yahoo.com

Published 17 July 2015.

This manuscript is generously published free of charge by ISASS, the International Society for the Advancement of Spine Surgery. Copyright $\odot 2015$ ISASS. To see more or order reprints or permissions, see http://ijssurgery.com. 